\title{
INCIDENCE OF NON-CANDIDA ALBICANS IN PATIENTS WITH URINARY TRACT INFECTION WITH SPECIAL REFERENCE TO SPECIATION AND ANTIFUNGAL SUSCEPTIBILITY
}

Ragini Ananth Kashid, Sandhya Belawadi, Gayatri Devi, Indumati

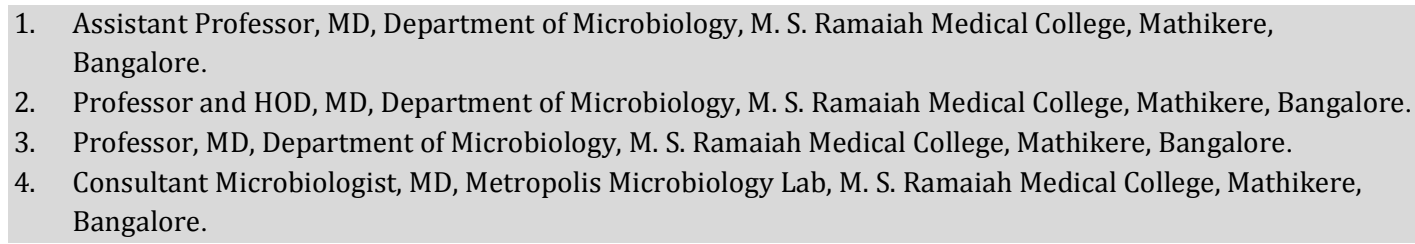

\section{CORRESPONDING AUTHOR}

Dr. Ragini Ananth Kashid, M.B.B.S. M.D. (Microbiology)

Flat No. A-601, Vasundhara Ashwini Apartments,

No. 14, Ring Road, Banashankari II stage

Bangalore 560070,

E-mail: ragini_rajgopal@hotmail.com,

Ph: 00919620380002.

ABSTRACT: BACKGROUND AND OBJECTIVES: Fungal urinary tract infections have become frequent, as a result of increased use of broad spectrum antibiotics, corticosteroids, immunosuppressive drugs and bladder catheters in acute care settings. The associated risk factors which are seen in cases of candiduria are: antibiotic therapy, female gender, urinary catheterization, surgical procedure and extended hospitalization. Candiduria has become a potential source of morbidity and mortality if untreated. We undertook a prospective study to note the incidence of non-Candida albicans in patients with urinary tract infection with special reference to speciation, antifungal susceptibility and the associated risk factors. METHODS: Candida species isolated from urine samples of patients with urinary tract infection were subjected to speciation using standard yeast identification protocol and CHROM agar. Antifungal Susceptibility testing was done by the disc diffusion method to amphotericin B and fluconazole. Clinical details and risk factors of the patients were noted down. RESULTS: Among the 60 culture positive cases, six Candida species which were isolated are : C.tropicalis (66.66\%), C.albicans (13.33\%), C.parapsilosis (8.33\%), C.glabrata (6.66\%), C.kefyr (3.33\%) and C.guilliermondii $(1.66 \%)$ The susceptibility pattern showed, that of the 60 isolates, $40 \%$ were resistant to fluconazole. No resistance was seen to amphotericin B. CONCLUSION: Isolation of non-Candida albicans species was more than Candida albicans. Candida tropicalis was the predominant isolate. The following risk factors were noted: $43.33 \%$ of the patients had diabetes mellitus, 30\%had history of prolonged antibiotics (cephalosporin and aminoglycosides), $16.66 \%$ had underlying renal pathology, $3.33 \%$ had post -renal transplant status, $1.66 \%$ were on steroids, $1.66 \%$ had pregnancy and $3.33 \%$ had no identifiable risk factors. $20 \%$ patients had an indwelling catheter in them. The antifungal resistance was more in the non-Candida albicans species than in Candida albicans. Hence there is a need for speciation, susceptibility testing of Candida species.

KEY WORDS: Candiduria, antifungal susceptibility testing, Candida speciation, CHROM agar, Non-Candida albicans, risk factors. 
INTRODUCTION: The significance of the presence of fungi in urine is not clearly understood. Fungal growth may represent contamination, bladder colonization, and primary infection or disseminated candidiasis. ${ }^{1}$ Fungal urinary tract infections have become frequent as a result of increased use of broad spectrum antibiotics, corticosteroids, immunosuppressive drugs and bladder catheters in acute care settings. ${ }^{2}$ The associated risk factors which are seen in cases of candiduria are: antibiotic therapy, female gender, urinary catheterization, surgical procedures and extended hospitalization. 3, 4, 5 Candiduria has become a potential source of morbidity and mortality if untreated. ${ }^{6}$ We undertook a prospective study to note the incidence of non-Candida albicans in patients with urinary tract infection with special reference to speciation, antifungal susceptibility and the associated risk factors.

MATERIALS AND METHODS: A prospective study, for a period of one year, was conducted in the Department of Microbiology, in which all patients who presented with signs and symptoms of urinary tract infection (bladder discomfort, low grade fever, and leucocytosis) were included in the study. The urine samples from these patients were collected with standard aseptic precautions. Information was collected, on the method in which the urine sample was collected(clean catch, suprapubic, urinary catheter).Data such as age, sex, prolonged antibiotic therapy, antifungal therapy and other possible risk factors were noted. The urine samples were processed within 2-4 hours. Candiduria was diagnosed by urine culture. The urine samples were spread by calibrated loop $(0.01 \mathrm{ml})$ as per standard protocol for urine culture. Plates were incubated aerobically at $37^{\circ} \mathrm{C}$ and read at 24 hours. Candida species isolated on culture plates, with a colony count $>1000$ colonies $/ \mathrm{ml}$, were considered indicate an active UTI. 6

The Candida isolates thus isolated, were identified to species level ,based on Grams Stain, KOH mount, India ink preparation, Culture on Sabouraud's Dextrose Agar, Germ Tube test, Urea hydrolysis, Cornmeal agar morphology (Dalmau technique), Sugar Fermentation Test, Sugar assimilation test (Auxanographic Plate Method ) 8,9 and CHROM Agar morphology.10,11

ANTIFUNGAL SUSCEPTIBILITY TEST :( Disc Diffusion test by Shadomy Ingroff method) was done, using Fluconazole $(10 \mu \mathrm{g})$ and Amphotericin -B (100 units) discs. The inoculum was standardized to 0.5 Mc Farland units. Candida albicans ATCC 90028 was used as control. 12, 13,14 The antifungal discs and the dehydrated culture media were procured from Hi- Media, Mumbai.

STATISTICAL ANALYSIS: Descriptive statistics were used to summarize continuous measures. The data was evaluated using $2 \times 2$ contingency tables using chi -square test. P values $<0.05$ were considered to be significant.

RESULTS: In a period of one year, a total of 4380 urine specimens were processed in the Microbiology Laboratory, of which 60 urine samples yielded Candida species. Of these 60 isolates, 48 isolates had been isolated where the specimen was collected by midstream clean catch urine specimen and 12 by indwelling catheter. In this study, there was no urine sample collected by suprapubic puncture method.

The isolation rate of Candida species from urine samples was $1.37 \%$

$65 \%(39 / 60)$ Candida isolates were isolated from males and $35 \%(21 / 60)$ from females. Among the 60 Candida isolates the species wise distribution was as follows: $66.66 \%$ ( 40/60) were C.tropicalis, $13.33 \%$ ( 8/60) were C. albicans, $8.33 \%(5 / 60)$ were C. parapsilosis, 
$6.66 \%$ (4/60) C. glabrata, 3.33\% (2/60) C. kefyr and 1.66\% (1/60) were C. guilliermondii. Refer Table 1.The isolation rate of non- Candida albicans species from urine samples was $1.18 \%$

The following risk factors were noted: $43.33 \%$ (26/60) of the patients had diabetes mellitus, $30 \%(18 / 60)$ had history of prolonged antibiotics (cephalosporin and aminoglycosides), $16.66 \%(10 / 60)$ had underlying renal pathology, 3.33\%(2/60) had post renal transplant status, $1.66 \%(1 / 60)$ were on steroids, $1.66 \%(1 / 60)$ had pregnancy and $3.33 \%(2 / 60)$ had no identifiable risk factors.20\% (12/60) patients had an indwelling catheter in them. Refer Table 2.

In the group of patients that had Diabetes mellitus as a risk factor, $61.53 \%(16 / 26)$ of the isolates were C. tropicalis, $11.53 \%(3 / 26)$ were C. albicans, $11.53 \%(3 / 26)$ were C. glabrata, $11.53 \%(3 / 26)$ C. parapsilosis and 3.84\% (1/26) of C. kefyr.

In the group that had prolonged medication as a risk factor, $77.77 \%(14 / 18)$ were $\mathrm{C}$. tropicalis, $11.11 \%$ (2/18) each were C. albicans and C. parapsilosis.

Among the 10 culture positive cases, of candiduria in patients with underlying renal pathology (other than complications of diabetic nephropathy) $70 \%(7 / 10)$ were C. tropicalis 10 $\%(1 / 10)$ each of C. albicans, C. glabrata and C. kefyr.

Among the 2 culture positive cases from post renal transplant patients, all 2 (100\%) were C. tropicalis.

ANTIFUNGAL SUSCEPTIBILITY TESTING: When tested for Fluconazole, it was observed that of the 8 isolates of $\mathrm{C}$. albicans, $3(37.5 \%)$ were resistant. Of the 52, non-Candida albicans isolates, $21(40.38 \%)$ were resistant to Fluconazole. No resistance was seen to Amphotericin B (Table 3).

DISCUSSION: Candiduria is the presence of yeast cells in urine and is an increasingly common finding in hospital patients. ${ }^{15}$ Candida spp. is often isolated from urine specimens and has been estimated to account for as many as $21 \%$ of urinary yeast isolates. ${ }^{16}$ Candiduria is a marker for haematogenous seeding to the kidneys. Candiduria reflects colonization or infection of the lower urinary tract or collecting system of the kidneys. ${ }^{15}$ In one study conducted by Lenner T, it was noted that $90 \%$ of patients with disseminated candidiasis who died, had renal involvement at autopsy. ${ }^{17}$ Candida species is the fifth most common nosocomial urinary pathogen in India. ${ }^{18}$ In another study by Mirdha B.R.et al 1998 the age range for funguria was 1-75 years. ${ }^{2}$

In a study on candiduria conducted by Artiago Kobayashi, 2004, Brazil, it was noted that $57.8 \%$ of female patients had candiduria. ${ }^{1}$ In contrast two Indian studies one by Mirdha B.R. et al. and in the Chakrabarthi A.et al., observed that $74.9 \%$ and $74.7 \%$ subjects with candiduria were males. ${ }^{2}, 19$ In our study $65 \%$ cases of candiduria were males (This could be an incidental finding in our study, as the number of males are more in the study).

Urinary tract candidiasis and candiduria is seen in association with disseminated candidiasis, diabetes mellitus, pregnancy, administration of antibiotics and unclean catheters. ${ }^{15}$, 20 The risk to develop candiduria increases by 12 fold after urinary catheterization, 6 fold after the use of broad spectrum antibiotics and urinary tract abnormalities, 4 fold following abdominal surgeries, 2 fold in the presence of diabetes mellitus and 1 fold in association with corticosteroid administration. However, no significant difference was found between the compared groups in terms of female sex and age. ${ }^{21}$

Catheterization can cause infection by introducing organisms during catheterization process or by allowing migration of the organisms into the bladder along the surface of the 
catheter from the external periurethral surfaces..$^{22}$

Diabetics are at an increase risk of fungal UTI. Diabetes mellitus may predispose patients to fungal candiduria by predisposing them to Candida colonization of vulvovestibular area (in women) by enhancing urinary fungal growth in the presence of glycosuria by lowering host resistance to invasion by Candida species as a consequence of impaired phagocytic activity and promoting stasis of urine in a neurogenic bladder. Guze and Harley observed that more than half the patients with funguria had diabetes mellitus. ${ }^{17}$ In the present study, $43.33 \%$ had diabetes mellitus as the risk factor.

Candiduria has become a potential source of morbidity and mortality if left untreated. It has been associated with $20 \%$ mortality in patients with urological pathology who require surgery. ${ }^{2}$ The present study had 10 cases of candiduria with underlying renal pathology other than diabetic nephropathy and its complications. Of these 10 cases, $6(60 \%)$ cases of benign prostatic hypertrophy, 1 each (10\%) of fungal cystitis, pelvi- uretero junction block, renal failure and posterior urethral valve.

Chakrabarthi A.et al (1999) studying nosocomial candidaemia identified immunosuppressive therapy in organ transplant recipients as a risk factor for acquiring infection with Candida species. ${ }^{22}$ In 2 cases of post renal transplant patients, who were on immunosuppressive therapy, Candida tropicalis was isolated from urine.

Prasad et al. state that isolation of non- Candida albicans spp. was higher (52.4\%) as compared to C. albicans (47.6\%). ${ }^{18}$ Chakrabarthi A et al found C. tropicalis was the commonest species isolated from urine $(58.7 \%)$ followed by C. albicans. ${ }^{19}$ In our study too, C. tropicalis was isolated more frequently than C. albicans.

ANTIFUNGAL SUSCEPTIBILITY TESTING: We adopted the Disc diffusion method as it is simple and easy to perform. But to confirm resistance of any strain, broth dilution test should be used and disc diffusion method can be used for preliminary screening.

The antifungal susceptibility pattern showed that $40 \%$ of the Candida isolates were resistant to Fluconazole. No resistance was seen for Amphotericin B.

From the data of the antifungal susceptibility testing, it was observed that resistance to Fluconazole was more in the non-Candida albicans group $(\mathrm{p}<0.05)$ when compared to $\mathrm{C}$. albicans. (Refer Table 3 and Results section).

This study mainly intended to define the spectrum of the various non-Candida albicans species and their antifungal susceptibility pattern, in cases of urinary tract infection. Mortality with candiduria is high in debilitated patients and with those with advanced age. In view of antifungal resistance being reported, identification of Candida species and their antifungal susceptibility pattern, is necessary to decide on the on the choice of antifungal drugs.

Table 1: Distribution of Candida specie

\begin{tabular}{|lcc|}
\hline \multicolumn{1}{|c}{ Candida species } & Number $\mathbf{n = 6 0}$ & Percentage\% \\
\hline C. tropicalis & 40 & $\mathbf{6 6 . 6 6}$ \\
C. albicans & 8 & $\mathbf{1 3 . 3 3}$ \\
C. parapsilosis & 5 & $\mathbf{0 8 . 3 3}$ \\
C. glabrata & 4 & $\mathbf{0 6 . 6 6}$ \\
C. kefyr & 2 & $\mathbf{0 3 . 3 3}$ \\
C. guilliermondii & 1 & $\mathbf{0 1 . 6 6}$ \\
\hline
\end{tabular}

Journal of Evolution of Medical and Dental Sciences/Volume1/ Issue4/October - 2012 Page 575 
Table 2: Associated risk factors

\begin{tabular}{|lcc|}
\hline Risk factor & Number $\mathbf{~} \mathbf{n} \mathbf{6 0}$ & Percentage $\%$ \\
\hline Diabetes mellitus & 26 & 43.33 \\
Broad spectrum antibiotics & 18 & 30.00 \\
Underlying renal pathology & 10 & 16.66 \\
Post-renal transplant status & 02 & 03.33 \\
Steroids & 01 & 01.66 \\
Pregnancy & 01 & 01.66 \\
No identifiable risk factor & 02 & 03.33 \\
\hline
\end{tabular}

Table 3: Antifungal susceptibility test

\begin{tabular}{|c|c|c|c|c|c|c|}
\hline Species & $\begin{array}{l}\text { Flucona } \\
\text { zole } \\
\text { Sensitiv } \\
\text { e }\end{array}$ & $\begin{array}{l}\text { Flucona } \\
\text { zole } \\
\text { Interme } \\
\text { diate }\end{array}$ & $\begin{array}{l}\text { Fluconaz } \\
\text { ole } \\
\text { Resistant }\end{array}$ & $\begin{array}{l}\text { Amphoterici } \\
\text { n B } \\
\text { Sensitive }\end{array}$ & $\begin{array}{l}\text { Amphoterici } \\
\mathrm{n} \mathrm{B} \\
\text { Intermediat } \\
\mathrm{e}\end{array}$ & $\begin{array}{l}\text { Amphoteri } \\
\text { cin B } \\
\text { Resistant }\end{array}$ \\
\hline C.tropicalis(40) & 23 & 0 & 17 & 40 & 0 & 0 \\
\hline C. albicans(8) & 5 & 0 & 3 & 8 & 0 & 0 \\
\hline C.parapsilosis(5) & 4 & 0 & 1 & 5 & 0 & 0 \\
\hline C. glabrata (4) & 3 & 0 & 1 & 4 & 0 & 0 \\
\hline C. kefyr(2) & 0 & 0 & 2 & 2 & 0 & 0 \\
\hline C.guilliermondii(1) & 1 & 0 & 0 & 1 & 0 & 0 \\
\hline
\end{tabular}

\section{REFERENCES:}

1. Kobayashi A, Fernandes L, Miranda C, Sousa D, Silva R. Candiduria in hospital patients: A study prospective. Mycopathologia 2004; 158: 49-52.

2. Mirdha BR, Sethi S, Banerjee U. Prevalence of fungal species in patients with funguria. Indian J of Med Res. 1998; 107:90-93.

3. Sobel JD. Management of asymptomatic candiduria. Int J Antimicrob Agents. 1999; 11: 285-88.

4. Lundstorm T, Sobel J. Nosocomial candiduria: A review. Clin Infect Dis 2001; 32: 1602-07.

5. Sobel JD, Vaquez JA. Fungal infections of the urinary tract. World J Urol. 1999; 17: 410 14.

6. Ang BSP, Talenti A, King B, Steekelberg JM, Wilson WR. Candidaemia from a urinary tract source: Microbiological aspects and clinical significance. Clin Infect Dis. 1993; 17 (4): 626-66.

7. Bukhary ZA. Candiduria: A review of clinical significance and Management. Saudi J Kidney Dis Transpl. 2008; 19: 350-60.

8. National workshop in Medical Mycology.29th Annual Congress of Indian Association of Medical Microbiologists. MICROCON 2005. Medical Mycology Laboratory Procedures. Sri Ramachandra Medical College and Research Institute, Porur, Chennai. Oct 2005. 
9. Workshop on fungal infections of eye, ear and skin: A laboratory perspective, Department of Microbiology, St. Johns Medical College, Bangalore. 2005; 1-15.

10. Odds FC, Bernaerts R. CHROMagar Candida, a new differential isolation medium for presumptive identification of clinically important Candida species. J Clin Microbiol. Aug 1994; 32(8): 1923-29.

11. Horvath L, Hospenthal, Murray KC, Dooley PD. Direct isolation of Candida species from blood cultures on the chromogenic medium CHROMagar Candida. J of Clin Microbiol. 2003; 41: 2629-32.

12. National Committee for Clinical Laboratory Standards. 2004. Method for antifungal disk diffusion susceptibility for yeasts. Approved guideline M44-A. National Committee for Clinical Laboratory Standards, Wayne, Pa.

13. Chakrabarthi A, Ghosh A, Kanta A, Kumar P. In vitro antifungal susceptibility of Candida. Indian J of Med Res. 1995; 102:13-19.

14. Chakrabarthi A, Mohan B, Shrivastava SK, Marak RSK, Ghosh A, Ray P. Change in the distribution and antifungal susceptibility of Candida species isolated from candidaemia cases in a tertiary care centre during 1996-2000. Indian J of Med Res. 2002; 116:5-12.

15. Chander Jagdish. Candidiasis. Textbook of Medical Mycology, $3^{\text {rd }}$ ed: New Delhi: Mehta Publishers; 2002. p. 266-90.

16. Sumangala BM. Candidiasis. J of the Academy of Clin Microbiologists 2004; 6(15): 6769.

17. Vazquez JA, Sobel SD. Mucosal candidiasis. Inf Dis Clinics North America 2002; 793820.

18. Prasad KN, et al. Role of yeasts as nosocomial pathogens and their susceptibility to Fluconazole and Amphotericin. Ind J of Med Res. 1999; 110:11-17.

19. Chakrabarthi A, Reddy TCS, Singhi S. Does Candiduria predict candidaemia? Ind J of Med Res. 1997; 106; 513-16.

20. Rippon JW. Medical Mycology.3rd ed. Philadelphia: W.B. Saunders

21. Bukhary ZA. Candiduria: A review of Clinical significance and management. Saudi J Kidney Dis Transpl. 2008; 19: 350 - 60.

22. Chakrabarthi, Singh K, Das S. Changing face of nosocomial candidaemia. Ind J of Med Microbiol. 1999; 17(78): 160-66. 